S. Kwok, M. Dopita, and R. Sutherland, eds.

\title{
Far-Ultraviolet Spectra of Planetary Nebulae
}

\author{
George Sonneborn \\ Laboratory for Astronomy and Solar Physics \\ NASA Goddard Space Flight Center \\ Greenbelt, Maryland 20771, USA
}

\begin{abstract}
The Far Ultraviolet Spectroscopic Explorer (FUSE) satellite provides a unique opportunity to obtain high-resolution far-UV spectra of a wide variety of astronomical objects, including planetary nebulae. Most FUSE observations of $\mathrm{PNe}$ to date have concentrated on the hot central star, providing a very effective way to study the atmosphere of the central star, the surrounding nebula through the absorption features from circumstellar gas. FUSE has found evidence of hot molecular hydrogen in several planetary nebulae, including M27 and $\mathrm{BD}+30^{\circ} 3639$. Central star spectra also reveal new information about stellar winds, mass loss, and photospheric abundances.
\end{abstract}

\section{Introduction}

The Far Ultraviolet Spectroscopic Explorer ( $F U S E$ ) provides the opportunity to obtain high-resolution far-UV spectra $(910-1180 \AA, \lambda / \Delta \lambda \sim 20,000)$ of a wide variety of planetary nebulae. The mission is described by Moos et al. (2000) and Sahnow et al. (2000). Most FUSE observations of planetary nebulae (PNe) to date have concentrated on the hot central star, providing a very effective way to study the surrounding nebula through the absorption features from the circumstellar gas as well as properties of the star as well. By selecting PNe with sufficient radial velocity, the circumstellar and interstellar components may be readily resolved.

This paper presents current work by FUSE observers in two areas: absorption spectroscopy of the nebula, in particular molecular hydrogen, and the stellar winds and mass loss from the central star. The intrinsic far-UV spectra of central stars and photospheric abundances, including evidence from FUSE spectra for an iron abundance deficiency in some PG1159 stars are described in this volume by Werner (2002).

\section{Molecular Hydrogen}

FUSE provides detailed information on the $\mathrm{H}_{2}$ populations in the excited rotational levels of the ground vibrational state because of the large number of 
absorption lines, with a wide range of intrinsic strengths (f-values), in its bandpass.

Vibrationally excited near-infrared transitions of $\mathrm{H}_{2}$ have been seen in dozens of planetary nebulae. The physical interpretation of this emission requires identification of the responsible excitation mechanism: possibilities include both collisional and radiative processes. Although the lowest excited vibrational levels of the ground electronic state, $v^{\prime \prime}=1,2$, can relatively easily be populated by collisions from $v^{\prime \prime}=0$, to significantly populate $v^{\prime \prime} \geq 3$ requires a fluorescence process involving "pumping" by UV absorptions in the $\mathrm{H}_{2}$ Lyman and Werner bands, followed by a UV plus IR radiative cascade. FUSE observations described below directly observed the lines responsible for the pumping, $\mathrm{H}_{2}$ in absorption against the UV spectrum of the central star. Several FUSE GI programs were designed to search for such nebular components of the $\mathrm{H}_{2}$ Lyman and Werner lines in several PNe which meet these criteria. In addition to strong $v^{\prime \prime}=0$ lines, there are also a few weak $v^{\prime \prime}=1$ and 2 transitions towards the long-wavelength end of the FUSE band, but the main spectral forest of lines from $v^{\prime \prime} \geq 3$ lies in the 1150-1500 $\AA$ region, which is better covered by STIS on HST (Meyer et al. 2001). Thus, STIS observations can be highly complementary to FUSE for $\mathrm{H}_{2}$ studies.

\subsection{Hot $\mathrm{H}_{2}$ in $\mathrm{M27}$}

An observation of the central star of M27 (NGC 6853, the "Dumbbell" nebula) was obtained early in the FUSE mission and analyzed by McCandliss (2001). The central star is extremely hot $\left(\mathrm{T}_{\text {eff }} \sim 140,000 \mathrm{~K}\right.$, Bohlin et al. 1982) and has two $\mathrm{H}_{2}$ absorption systems separated by $\sim 42 \mathrm{~km} \mathrm{~s}^{-1}$, with distinctly different ground state populations. The blue component $\left(v_{r}=-69 \mathrm{~km} \mathrm{~s}^{-1}\right)$ shows lines originating from a highly excited ground state, with rotational levels $\left(J^{\prime \prime}\right)$ up to 15 and vibrational levels $\left(v^{\prime \prime}\right)$ at least as high as 2 . This unusually rich $\mathrm{H}_{2}$ spectrum has over $1000 \mathrm{H}_{2}$ lines in this component. Many of the absorption lines originating from $v^{\prime \prime}=1$ and 2 are located longward of the $\left(v^{\prime}-v^{\prime \prime}=0-0\right)$ band head at $1108.12 \AA$, and serve as unambiguous markers of hot $\mathrm{H}_{2}$. The redward component $\left(v_{r}=-27 \mathrm{~km} \mathrm{~s}^{-1}\right)$ is much cooler $\left(J^{\prime \prime}{ }_{\max }=5, v^{\prime \prime}=0\right)$ and may be associated with foreground gas. However, the separation of the hot and cool components is within the velocity separations observed by Goudis et al. (1978) in Fabry-Perot interferograms of O I, N II, and O III emission lines. It is possible that the cool component is also associated with the nebula.

McCandliss (2001) finds $\log \left(N\left(\mathrm{H}_{2}\right)\right)=16.6 \mathrm{~cm}^{-2}, \mathrm{~b}=5 \mathrm{~km} \mathrm{~s}^{-1}$, and $T \sim 2400 \mathrm{~K}$ for the blue component. The red component gives $\log \left(N\left(\mathrm{H}_{2}\right)\right)$ $=18.0 \mathrm{~cm}^{-2}, \mathrm{~b}=2 \mathrm{~km} \mathrm{~s}^{-1}$, and $T \sim 200 \mathrm{~K}$. A small portion of the spectrum is shown in Figure 1 and compared with a synthetic spectrum computed with these parameters. Figure 2 show the curve of growth (COG) and excitation for the hot component.

Judging from the present analysis, shock excitation of $\mathrm{H}_{2}$ in M27 is the preferred mechanism over fluorescent excitation, in spite the presence of the very hot central star. Shock excitation is consistent with IR emission diagnostics (Zuckerman \& Gatley, 1988). In addition, no UV fluorescence emission was detected from the nebula in a far-UV long-slit spectrum of M27 obtained on a NASA-JHU sounding rocket flight (McCandliss 2001). 


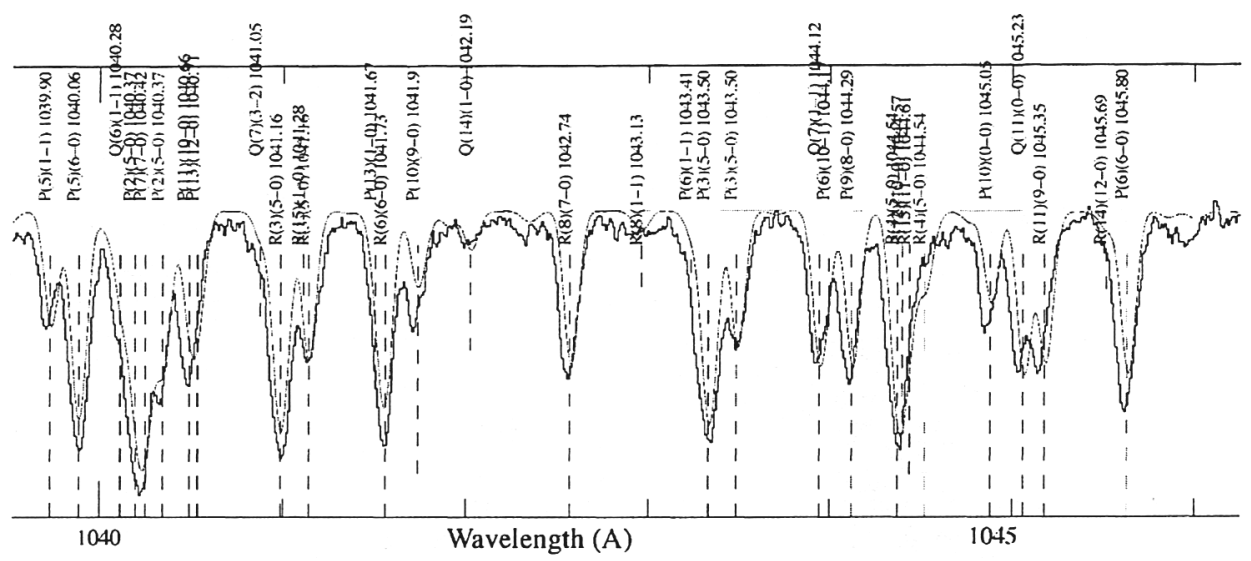

Figure 1. FUSE 1040 - $1046 \AA$ spectrum (histogram line) of the central star of M27 is shown along with the model described in the text (smooth line). All the identified spectral features are $\mathrm{H}_{2}$. The transition and rest wavelength are given for each $\mathrm{H}_{2}$ feature fit in the model.
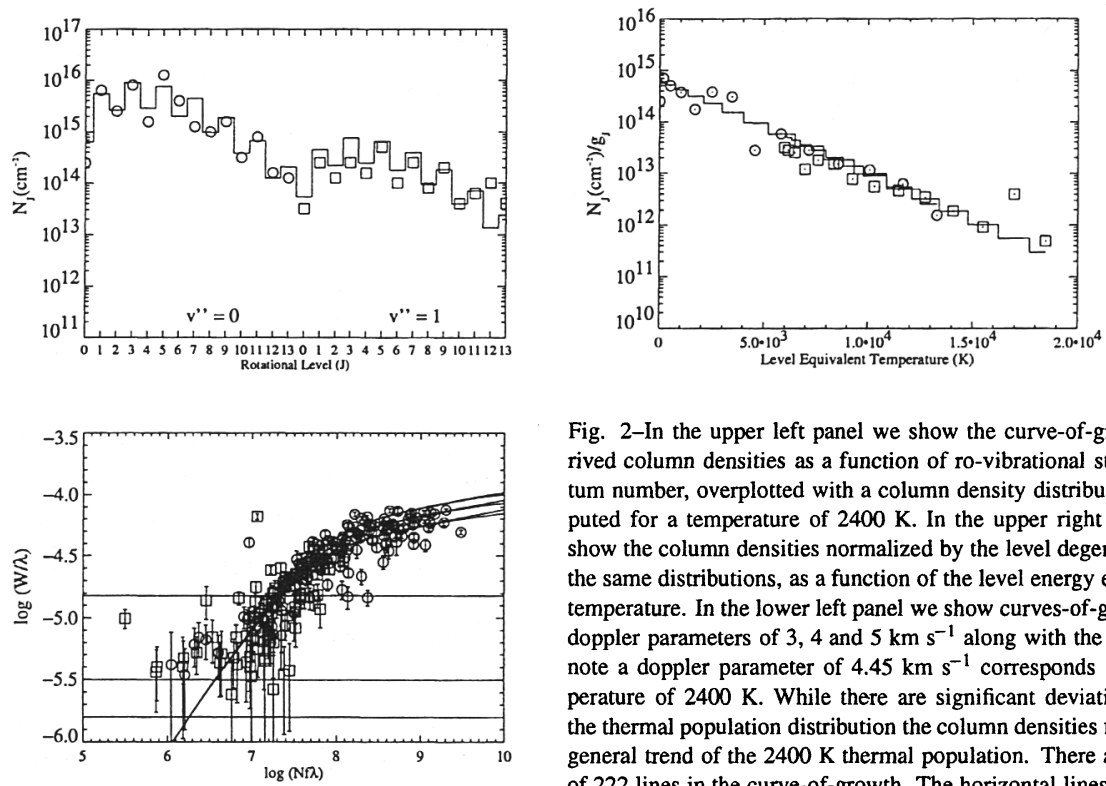

Fig. 2-In the upper left panel we show the curve-of-growth derived column densities as a function of ro-vibrational state quantum number, overplotted with a column density distribution computed for a temperature of $2400 \mathrm{~K}$. In the upper right panel we show the column densities normalized by the level degeneracy for the same distributions, as a function of the level energy equivalent temperature. In the lower left panel we show curves-of-growth for doppler parameters of 3,4 and $5 \mathrm{~km} \mathrm{~s}^{-1}$ along with the data. We note a doppler parameter of $4.45 \mathrm{~km} \mathrm{~s}^{-1}$ corresponds to a temperature of $2400 \mathrm{~K}$. While there are significant deviations from the thermal population distribution the column densities follow the general trend of the $2400 \mathrm{~K}$ thermal population. There are a total of 222 lines in the curve-of-growth. The horizontal lines mark the extrema and mean of the equivalent width errors.

Figure 2. Curve of growth analysis for the hot $\mathrm{H}_{2}$ component found in the FUSE spectrum of the central star of M27. 


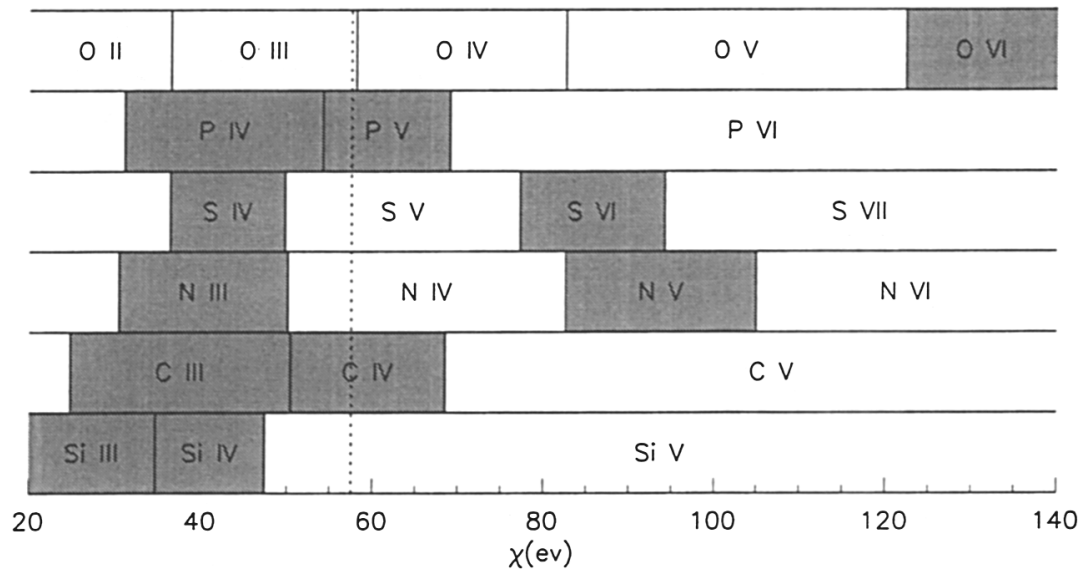

Figure 3. Ionization stages as a function of ionization potential for abundant elements. Stages with resonance lines observable by FUSE and $H S T$ are shaded. The other stages are unobservable (no strong lines longward of the Lyman limit). The vertical dashed line at $\sim 58$ $\mathrm{eV}$ marks the dominant ionization stage in $\mathrm{O}$ star winds.

\section{2. $\quad \mathrm{BD}+303639$}

Dinerstein, Bowers, Jenkins, \& Draine (2002) have analyzed far-UV lines of $\mathrm{H}_{2}$ observed by both FUSE and STIS, for $\mathrm{BD}+30^{\circ} 3639$. This compact young PN displays multiple velocity components (separated by $\sim 50 \mathrm{~km} \mathrm{~s}^{-1}$ ) in absorption lines from many excited rotational and vibrational levels. $\mathrm{BD}+30^{\circ} 3639$ is a source of near-IR $\mathrm{H}_{2}$ line emission, although the IR emission peaks several arc seconds east of the central star (Shupe et al. 1998). It must be kept in mind that the UV absorption method samples a pencil beam toward the star, a different line of sight than the IR peak, and therefore may sample different physical conditions.

For $\mathrm{BD}+30^{\circ} 3639$, as well as other PN central stars with strong winds, some $\mathrm{H}_{2}$ lines fall in deep minima of stellar P-Cygni line profiles and are unmeasurable, but usually there are more accessible lines from the same levels in other Lyman or Werner bands. This redundancy, of course, underlies the equivalent width/COG method of analysis for lines that are partially or strongly saturated. Dinerstein, et al. (2002) use both COG and component-fitting techniques to derive column densities for many levels, finding values of $\sim$ few $\times 10^{13} \mathrm{~cm}^{-2}$ for vibrational levels as high as $v^{\prime \prime}=7$ and rotational levels up to $J^{\prime \prime}=5$ (note that odd- 
valued J-levels enjoy the "ortho" factor-of-three statistical weight advantage). On the other hand, populations for $v^{\prime \prime}=0, J^{\prime \prime}=2,3,4$, and 5, are two orders of magnitude higher, $\sim$ few $\times 10^{15} \mathrm{~cm}^{-2}$ per level. This pattern of populations, with a relatively flat tail extending to high $v^{\prime \prime}$ values, is a clear indicator that a radiative excitation mechanism is operating. $\mathrm{BD}+30^{\circ} 3639$ and similar sources offer a superb opportunity for detailed study of the UV-excitation of $\mathrm{H}_{2}$ a key process throughout the diffuse ISM wherever UV radiation illuminates molecular material.

\section{The Central Stars}

\subsection{Central Star Mass Loss}

Some PNe have very highly ionized stellar winds (e.g. strong O VI and no C IV) accompanied by high outflow velocities $\left(v>2000 \mathrm{~km} \mathrm{~s}^{-1}\right)$, for example, K116 observed by Werner (2002) and NGC 7009 observed by Iping \& Sonneborn (2002). These stars have very hot central stars $\left(T_{\text {eff }}>100,000 \mathrm{~K}\right)$. The presence of such strong, highly ionized winds in these stars may be correlated with the occurrence of X-ray emission from PNe. Uncertainties in knowledge of the ionization state in the wind and saturation effects in strong wind lines (e.g. C IV $\lambda \lambda 1548-1550, \mathrm{~N} V \lambda \lambda 1238-1242)$ have limited progress in modelling the winds and determining mass loss rates in many central stars. There are, however, good prospects for progress with improved models now that FUSE spectra are becoming available for many PN central stars.

Analysis of lines in the FUSE spectral range offers two reasons to expect considerable progress in this area. First, there are a large number of resonant transitions of species that are less abundant than $\mathrm{C}, \mathrm{N}$, and $\mathrm{O}$, hence their wind lines are unsaturated, or much less so, than the lines available at longer ultraviolet wavelengths. Second, there are a significant number of strong lines covering a wide range of ionization potential. These include O VI $\lambda \lambda 1032$ 1038, Si III $\lambda \lambda 1108-1113$, Si IV $\lambda \lambda 1120-1128$, C III $\lambda \lambda 977$, C III $\lambda 1175$, N III $\lambda 990$, S III $\lambda 1012$, S IV $\lambda \lambda 1063-1074$, S VI $\lambda \lambda$ 933-944, and P V $\lambda \lambda 11118-1128$. Figure 3 shows which ionization stages of abundant elements $(\mathrm{C}, \mathrm{N}, \mathrm{O}, \mathrm{Si}, \mathrm{S}$, and $\mathrm{P}$ ) have observable resonance lines longward of the Lyman limit. These lines are important for studying stellar winds and mass loss in $\mathrm{O}$ stars and PN central stars. Several commonly observed species (N V, S VI) are "super-ions" because they are an ionization stage above the dominant one for a given element. O VI is even more extreme in that it is two stages above the dominant stage.

The complete FUSE spectrum of NGC 7009 is shown in the paper by Iping \& Sonneborn (2002) in this conference. This observation was obtained using the $30 \times 30$ arcsec aperture, and includes the central star and almost all of the nebula. The strong continuum of the central star is punctuated by many absorption lines of molecular hydrogen and atomic species as well as by emission from the nebula within the aperture. The strong P-Cygni feature at 1025-1040 $\AA$ is O VI in the stellar wind of the central star. The extreme degree of ionization in the wind of this star, with the very strong OVI P-Cygni profile, is reinforced by the fact that IUE spectra (1200-3000 $\AA$ ) of this star show no wind lines, including C IV $\lambda \lambda 1548-1550$ and NV $\lambda \lambda 1238-1242$. Since the O VI wind feature is heavily saturated, it is useful only for determining the terminal velocity of the wind. 


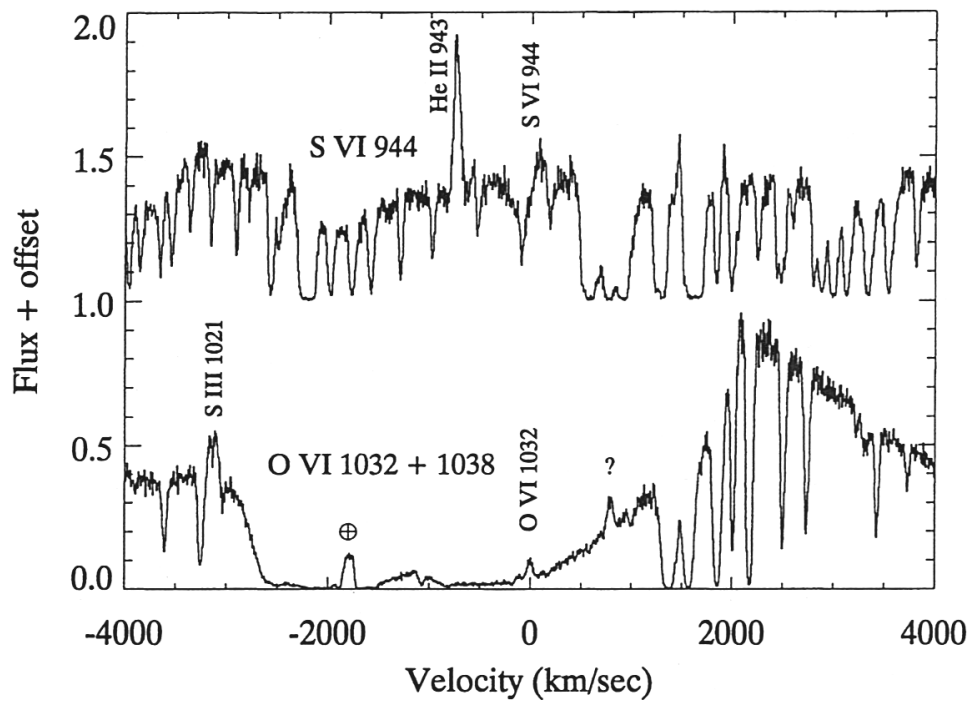

Figure 4. O VI $\lambda \lambda 1032-38$ and S VI $\lambda \lambda 933-44$ wind line profiles in NGC 7009. Most of the narrow absorption features are interstellar $\mathrm{H}_{2}$. He II, S II, and several other emission features are also shown.

However, the S VI lines have wind features that are far from saturation. These two profiles are shown in Figure 4.

Figure 5 shows the FUSE spectrum of Hen 2-274, an example of the type of spectrum from which state-of-the-art wind modelling for the central star should be possible. This PN has a central star with a much lower $T_{\text {eff }}$, similar to those found in O stars (Fullerton et al. 2000). This spectrum contains stellar lines from most if not all of the transitions listed at the beginning of $\S 3$. With a wide range of ionization potential, including multiple ionization stages for the same element, detailed models of the atmosphere and the wind of the central star should be possible. The spectrum of Hen 2-138 is another example of a PN central star with a similar stellar wind spectrum. This spectrum is shown by Iping \& Sonneborn (2002).

FUSE spectra are available publically from the FUSE archive six months after they are archived. The web site is http://archive.stsci.edu/fuse/.

Acknowledgments. The author is grateful H. Dinerstein, R. Iping, D. Massa, and S. McCandliss for permission to discuss their work prior to publication.

\section{References}

Bohlin, R. C., Harrington, J. P., \& Stecher, T. P., 1982, ApJ, 252, 635 

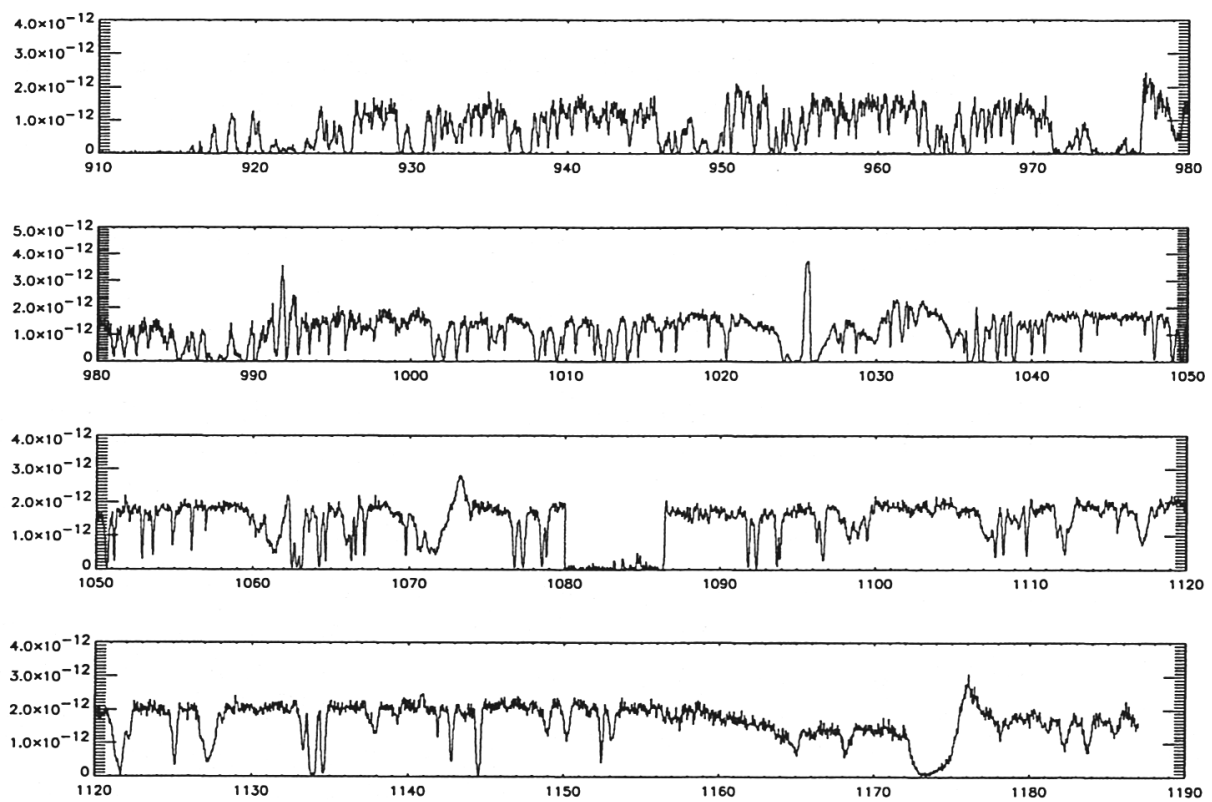

Figure 5. FUSE spectrum of Hen 2-274 from the Lyman limit to $1180 \AA$. The gap at $1080-1090 \AA$ is the result of a gap in spectral coverage in the $\mathrm{LiF}$ channels and the absence of the SiC2 channel for this particular observation.

Dinerstein, H., Bowers, C., Jenkins, E. B., \& Draine, B. T. 2000, in "Planetary Nebulae: Their Evolution and Role in the Universe," ed. M. Dopita \& R. Sutherland, A.S.P. Conf. Ser. (this volume)

Fullerton, A. W., et al., 2000, ApJ, 538, L43

Goudis, C. et al. 1978, MNRAS, 182, 13

Iping, R. C., \& Sonneborn, G. 2002, in "Planetary Nebulae: Their Evolution and Role in the Universe," ed. M. Dopita \& R. Sutherland, A.S.P. Conf. Ser. (this volume)

McCandliss, S. R., 2001, in "The Challenge of High Resolution X-Ray Through IR Spectroscopy of Photoionized Plasmas", ed. G. Ferland, ASP Conf. Ser. (in press)

Meyer, D., et al. 2001, ApJ, 553, L59

Moos, H. W., et al. 2000, ApJ, 538, L1

Sahnow, D. J., et al. 2000, ApJ538, L7

Shupe, D. L. et al. 1998, AJ, 498, 267

Werner, K. 2002, in "Planetary Nebulae: Their Evolution and Role in the Universe," ed. M. Dopita \& R. Sutherland, A.S.P. Conf. Ser. (this volume)

Zuckerman, B., \& Gatley, I., 1988, ApJ, 324, 501 